\title{
THE INFLUENCE OF THE SLIDE-TO-ROLL RATIO ON THE FRICTION COEFFICIENT AND FILM THICKNESS OF EHD POINT CONTACTS UNDER STEADY STATE AND TRANSIENT CONDITIONS
}

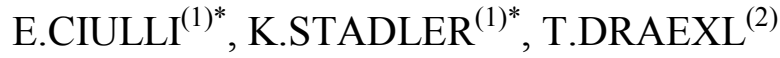 \\ ${ }^{(1)}$ Università di Pisa, Dipartimento di Ingegneria Meccanica Nucleare e della Produzione Via Diotisalvi 2, 56126 Pisa, \\ Italy \\ ciulli@ing.unipi.it, kenred.stadler@skf.com \\ ${ }^{(2)}$ Forschungsstelle fuer Zahnräder und Getriebebau, Lehrstuhl fuer Maschinenelemente, Technische Universität \\ München, Boltzmannstrasse 15, 85748 Garching, Germany \\ draexl@fzg.mw.tum.de \\ * corresponding authors
}

\begin{abstract}
The effect of different slide-to-roll ratios has been experimentally investigated under steady state and transient conditions using a steel ball in contact with the plane surface of a glass disc. Under transient conditions the entraining velocity has been varied with a sinusoidal law at two different frequencies. Measurements of the friction force and film thickness using optical interferometry have been made.

For the same working conditions, different friction coefficient trends found for positive and negative slide-to-roll ratios can be related to different film thickness values and shapes. The combination of different thermal effects could be a possible explanation for the obtained results.
\end{abstract}

Keywords: EHD film thickness, friction coefficient, thermal effects. 


\section{INTRODUCTION}

Many machine elements such as rolling element bearings, gears and cams are elastohydrodynamically lubricated (EHD). Some of these elements work under conditions where the effect of sliding is not negligible anymore. For pure rolling conditions, works of e.g. Grubin, Dowson and Higginson [1, 2] brought fundamental understanding of the working performance of machine elements with non-conformal lubricated contacts, by developing the Elastohydrodynamic lubrication theory (EHL). In addition, studies have been made for taking into account sliding effects. For instance Wilson [3] and Olver [4] developed formulas where the deviation from the pure rolling film thickness values can be calculated with a correction factor and explained by inlet heating, or a temperature rise can be calculated by the bulk and flash temperature theory. Smeeth and Spikes [5] tried to explain some of their experimental findings, obtained measuring the film thickness for different slide-to-roll-ratios (S) under steady state conditions, with the theory explained in $[3,4]$. However, the inlet heating and rise in bulk temperature could explain the change in film thickness by different S only to some extent. Further insights were gained with the experimental work of Kaneta [6]. He has shown the formation of a dimple in the central part of the contact at high sliding conditions if materials with different thermal properties are used. The classical theory, however, assumed a flat plateau in the central part of the contact and the film thickness and shape are mainly determined by the conditions at the contact entrance. In [7], Kaneta and Yang could verify the experimental findings with numerical simulations by solving also the energy equation and explaining the occurrence of dimples with the so-called temperature viscosity wedge effect.

So far most of these works used steady state condition in order to explain thermal effects caused in sliding contacts. But it must be considered that most machine elements work under transient conditions by means of changes in radius of curvature, load or speed. In some experimental and numerical works using transient conditions, such as the ones of Sugimura [8] or Zhao [9], the effect 
of squeeze on film thickness was studied. In Zhai [10] also the friction coefficient for a contact under mixed lubrication conditions was simulated. The effects of different values of S combined with a change in velocity, however, are rarely investigated.

This experimental work tries to contribute further insights into the field of EHD combining both effects, caused by transient conditions and different S.

In a previous work, [11], differences between the EHD friction coefficients obtained for $\mathrm{S}<0$ and $\mathrm{S}>0$ were found, particularly when the contacting materials had different thermal properties. For steady state as well as transient conditions, effects like an intersection in the friction coefficients for negative S (disc running faster than the specimen) were found when using a glass-on-steel contact. This could be explained by thermal effects like the temperature viscosity wedge action (dimple). However, further investigations were necessary. New tests have been carried out using a smaller steel ball at working conditions similar to the previous work. More attention is paid to analyzing the film thickness. Similar effects as reported in [11] have been found, like the intersection of the friction coefficient curves for a glass-on-steel contact when $\mathrm{S}<0$. By changing the speed with a sinusoidal law, some dependence of transient effects (film thickness loops) on S has been observed.

\section{TEST SET UP}

A picture of the test rig used is shown in Fig.1. A glass disc $(\mathrm{Rq}=0.01 \mu \mathrm{m})$ has been loaded against a bearing steel ball made of AISI52100 $(\mathrm{Rq}=0.007 \mu \mathrm{m})$ with a diameter $\mathrm{D}$ of $10.319 \mathrm{~mm}$.

In order to test for different $\mathrm{S}$, the specimen and the disc are driven separately by electric motors controlled through a LabView ${ }^{\circledR}$ program running on a standard PC. An oil stream is directly injected near the contact area that ensures fully flooded conditions and also a fast heat exchange. The actual oil temperature is measured by a thermocouple located close the contact. The load is applied via a lever mechanism supported on a radial gas bearing, whose axial motion is constrained by a load cell used to measure the friction force with a precision of $0.05 \mathrm{~N}$. An optical 
interferometry system with white light and a high-speed camera are used to measure the film thickness and shape. All data like the velocity of the disc and the specimen, as well as the friction force acting on the specimen, are recorded by the data acquisition system.

If not explicitly stated otherwise, the following test conditions can be assumed. The oil temperature and the load has been kept constant during all tests respectively at $\mathrm{T}=12^{\circ} \mathrm{C}$ and $\mathrm{F}=15 \mathrm{~N}$ (Hertzian pressure $\mathrm{p}_{\mathrm{z}}=0.7 \mathrm{GPa}$ ). A paraffin base mineral oil (SN500) has been used, whose dynamic viscosity, pressure viscosity coefficient and density are $\eta_{0}=0.497 \mathrm{~Pa} \cdot \mathrm{s}, \alpha=2.8710^{-8} \mathrm{~Pa}^{-1}$ and $\rho_{0}=892 \mathrm{~kg} / \mathrm{m}^{3}$ at test temperature and atmospheric pressure. The thermal conductivity and specific heat of the oil are $0.137 \mathrm{~W} / \mathrm{mK}$ and $1934 \mathrm{~J} / \mathrm{kgK}$ [12]. The contact solids have the following thermal properties: thermal conductivity: glass $0.78 \mathrm{~W} / \mathrm{mK}$, steel $46 \mathrm{~W} / \mathrm{mK}$, specific heat: glass $840 \mathrm{~J} / \mathrm{kg} \mathrm{K}$, steel $470 \mathrm{~J} / \mathrm{kg} \mathrm{K}$.

First, tests have been carried out under steady state conditions, for entraining velocities $u_{e}$ ranging from $u_{e}=0.025$ to $u_{e}=0.25 \mathrm{~m} / \mathrm{s}$ and different $S$ of $0, \pm 0.8$ and $\pm 1.8\left(u_{e}=\left(u_{s}+u_{d}\right) / 2\right.$ where $u_{s}$ and $u_{d}$ are the surface speeds of the steel ball and the disc; $\left.S=\left(u_{s}-u_{d}\right) / u_{e}\right)$.

Second, to create transient conditions, the entraining velocity has been varied with a sinusoidal law between $u_{\min }=0.04 \mathrm{~m} / \mathrm{s}$ and $u_{\max }=0.225 \mathrm{~m} / \mathrm{s}$ at two different test frequencies of $0.25 \mathrm{~Hz}$ and $1 \mathrm{~Hz}$. The values of $\mathrm{u}_{\mathrm{e}}$ and $\mathrm{S}$ have been chosen in a way to avoid velocity inversions and possible starvation effects.

\section{FRICTION MEASUREMENTS}

Some preliminary tests were carried out with a steel ball of diameter $\mathrm{D}=15.081 \mathrm{~mm}$ and a load of $\mathrm{F}=20 \mathrm{~N}\left(\mathrm{p}_{\mathrm{Hz}}=0.6 \mathrm{GPa}\right)$. In Fig. 2 it is worth to see some of the results for the friction coefficients obtained under steady state conditions.

Negative $\mathrm{S}$ signifies that the disc is running faster than the steel ball and vice versa, positive $\mathrm{S}$ signifies that the disc is running slower than the steel ball. For all the test conditions investigated we 
can assume fluid film lubrication conditions, which mean that the influence of the surface roughness is negligible. In fact, a reference lambda value, $\Lambda$, of three for pure rolling conditions was reached already for velocities less than $0.025 \mathrm{~m} / \mathrm{s}$ thanks to the relatively high oil viscosity and low roughness $(\Lambda=$ ratio between the minimum film thickness and the equivalent root mean square roughness of the two bodies in contact). As well known, fluid film lubrication conditions can be assumed at lambda values higher than 2-3 in EHD contacts.

As shown in Fig.2, a difference in the friction coefficient trends is found depending if $\mathrm{S}$ is negative or positive (the contacting materials have different thermal properties). For high $\mathrm{S}$ $(S= \pm 1.8)$, the friction coefficient curve decreases faster than for positive $S$. The intersection between $\mathrm{S}=-1.8$ and $\mathrm{S}=-0.8$ curves is caused by different thermal effects, as explained in section 5 .

Fig. 3 to Fig. 8 show the results of the friction coefficient measurements obtained with the steel ball of diameter $10.319 \mathrm{~mm}$ under steady state as well as transient conditions.

In Fig.3 the friction coefficient trends for negative values of S under steady state conditions are presented. For comparisons, the corresponding results under transient conditions for $\mathrm{f}=0.25 \mathrm{~Hz}$ and $\mathrm{f}=1 \mathrm{~Hz}$ are shown in Fig. 4 and Fig.5 respectively. Attention should be paid to the fact that the measurement range of the velocity is slightly different for transient conditions $\left(\mathrm{u}_{\min }=0.055 \mathrm{~m} / \mathrm{s}\right.$, $\left.\mathrm{u}_{\max }=0.225 \mathrm{~m} / \mathrm{s}\right)$.

However, looking at the corresponding velocities, the same effects described above are found under steady state conditions as well as under transient conditions. Under variable velocity conditions the thermal effect for $\mathrm{S}<0$ (intersection of the friction coefficient curves) is superimposed by transient effects, which means a friction loop develops instead of a single line. As expected, the size of the loops grows with increasing test frequency (Fig.5). The solid line in Fig.4 and Fig.5 represents the friction coefficient values under accelerating conditions and the dashed line under decelerating conditions. 
The corresponding friction coefficient values for positive S are presented in Fig.6 to Fig.8. Looking at Fig.6 the intersection of the friction coefficient trends is very small. Tests at higher velocities could give further insights, but due to the small specimen used some vibration problems arose at high rotational speeds (for small radii higher rpm are necessary to reach high surface velocities). However, for higher surface velocities, results were obtained in [11] using a larger steel ball of diameter $\mathrm{D}=41.275 \mathrm{~mm}$ and a different load $\left(\mathrm{F}=31 \mathrm{~N}, \mathrm{p}_{\mathrm{Hz}}=0.38 \mathrm{GPa}\right)$ but no intersection was found for positive $\mathrm{S}$.

\section{FILM THICKNESS MEASUREMENTS}

The film thickness and shape has been analyzed by converting the white light interferograms obtained in the RGB (Red, Green, and Blue) color space into the HSV (Hue, Saturation and Value) color space. After phase unwrapping the hue channel of the HSV images, the film thickness can be evaluated at every point by comparison with a calibration table. A Hertzian contact was used for calibration purpose. For a detailed explanation of this technique the reader is referred for example to [13].

In Fig.9 and Fig.10 some results of the central film thickness measured under steady state conditions for different S are shown. Fig.9 shows the central film thickness over the entraining velocity for negative and positive S. Especially at higher velocities it can be seen that the central film thickness $h_{c e n}$ is a little bit higher for negative $S$ than for positive $S$. The highest film thickness values, however, are always found under pure rolling conditions $(\mathrm{S}=0)$. This can be seen also in Fig.10, where the central film thickness is shown over $\mathrm{S}$ for four different entraining velocities.

For small $\mathrm{S}$ the film thickness changes only a little, whereas for higher $\mathrm{S}$ the film thickness reduces around $20-30 \%$ compared to pure rolling conditions. In addition, it appears that the film thickness diminishes more for positive than for negative $\mathrm{S}$; this effect decreases for low entraining velocities (see curve d in Fig.10). In [5] a similar behavior was found. 
In Fig.11 and Fig.12 the measured film thicknesses under transient conditions are shown. A plot of the film thickness over velocity at $1 \mathrm{~Hz}$ and $\mathrm{S}=0$ is shown in Fig.11a compared to the steady state results. Central and minimum film thicknesses for two frequencies are shown for different values of $\mathrm{S}$ in Fig.11b and Fig.12, where the measured points are not reported for clarity reasons. The gross lines refer to the central film thicknesses and the thin lines to the minimum film thicknesses. Furthermore the solid lines refer to the film thicknesses obtained at test frequency of $1 \mathrm{~Hz}$ and the dashed lines to the test frequency of $0.25 \mathrm{~Hz}$. For each curve higher film thicknesses correspond to decelerating speed and lower film thicknesses to accelerating speed (the arrows show the direction of the entraining speed variations). Looking at the friction loops under transient conditions presented before, one will see that a higher friction coefficient corresponds to a lower film thickness and vice versa a lower friction coefficient corresponds to a higher film thickness.

In Fig.11 and 12 several effects due to transient conditions and sliding (thermal effects) are coming together. Looking first to the diagrams of Fig.11 for pure rolling conditions, the influence of thermal effects due to sliding should be negligible (100\% pure rolling condition will be reached only in theory). As it can be seen, for the test frequency of $1 \mathrm{~Hz}$ (solid lines) the film thickness loops are bigger than for $0.25 \mathrm{~Hz}$ (dashed lines), similarly to the friction coefficient loops. Such effects were also found in $[8,14]$. Both the central and minimum film thicknesses are higher for decreasing velocity than under steady state conditions, while for increasing velocity they are lower. Due to the higher accelerations, this effect is more distinct for the test frequency of $1 \mathrm{~Hz}$ than for $0.25 \mathrm{~Hz}$. At $1 \mathrm{~Hz}$ this hysteresis behavior is so strong that the extreme values (maximum and minimum) of the film thicknesses do not coincide with the extremes of the entraining velocity. Instead, film thicknesses continue to increase while velocity is already decreasing and reach their maximum only a few measuring points later. When the entraining velocity passes its minimum, the same behavior can be observed correspondingly. 
Some effects similar to the ones found under steady state conditions for different S (Fig.10) can be recognized: independently on the test frequency the highest film thicknesses are reached for pure rolling ( $\mathrm{S}=0$ ) whereas for high $|\mathrm{S}|$ the film thicknesses are lower. In addition, also under transient conditions an asymmetry is found in the film thickness results by means of lower values for positive $\mathrm{S}$ as also evidenced in Fig.13 where film thickness and entraining velocity are plotted as a function of time. The asymmetric reduction in film thickness for three extreme slide-to-roll ratios as well as the shift between the central film thickness and the corresponding velocity can clearly be seen.

Furthermore, as shown in Fig.12, with increasing $S$ the "loop effect" diminishes. For $S=+1.8$ and $1 \mathrm{~Hz}$ the film thickness loops are smaller than the ones at $\mathrm{S}=+0.8$ and have nearly the same size like the loops for $0.25 \mathrm{~Hz}$. At a test frequency of $0.25 \mathrm{~Hz}$ the decrease of the loops is less pronounced because transient effects are smaller compared to $1 \mathrm{~Hz}$. Comparing $\mathrm{S}=-1.8$ at $1 \mathrm{~Hz}$ with $\mathrm{S}=+1.8$ a significant difference is found. For $\mathrm{S}=-1.8$ and $1 \mathrm{~Hz}$ a bigger film thickness loop is still evident. The results are discussed in the next sections.

\section{DISCUSSION}

\subsection{Friction coefficient}

Some explanations can be given for the intersection of the friction coefficients in Fig.3-5, considering the increase in oil temperature in contact depending on S. Also the temperature viscosity wedge action effect, which is caused by the variation of the temperature distribution across the oil film along the oil entrainment direction, has an influence.

First, because the oil temperature used in this work is relatively low, only a few degrees in temperature rise diminish significantly the viscosity of the oil. For example an increase in temperature from $\mathrm{T}=12^{\circ} \mathrm{C}$ to $\mathrm{T}=15^{\circ} \mathrm{C}$ reduces the dynamic viscosity by about $0.1 \mathrm{~Pa} \cdot \mathrm{s}$. The glass disc has a lower thermal conductivity than the steel ball. This means that the oil temperature close 
to the glass disc is higher and therefore the oil viscosity lower than close to the steel ball. For high negative values of S (glass disc running faster than the ball) the oil temperature will increase relatively fast. Furthermore, with increasing velocity the low viscosity oil is resisted strongly by the high viscosity oil close to the steel ball and a wedge and thus a dimple can be formed for higher velocities as shown in Fig. 14. For $S=-1.8$, starting from an entraining velocity of about $u_{e}=0.3 \mathrm{~m} / \mathrm{s}$, the effect of the temperature viscosity wedge is clearly visible as a dimple develops. For further descriptions with regard to dimples caused by the temperature viscosity wedge phenomenon, the reader is referred to $[7,11]$.

If a dimple occurs a significant increase in contact temperature is reported e.g. in $[15,16]$. This will lead to a lower viscosity and less shearing so that the hydrodynamic friction decreases faster for high negative $\mathrm{S}$ (see $\mathrm{S}=-1.8$, Fig.3-5). Even if no deep dimples have been found for the velocity range used, a small temperature viscosity wedge action effect could be sufficient in order to increase the temperature in contact by a few degrees Celsius. A small shadow before the outlet restriction shown in Fig. 14 at $\mathrm{u}_{\mathrm{e}}=0.15 \mathrm{~m} / \mathrm{s}$, as well as the local increase in the central film thickness for $S=-1.8$ shown in Fig.15, indicate the first stages in the development of a dimple. Besides, the local increase in the central film thickness might also reduce the effect of shearing [17]. The measurement of the temperature directly inside the contact was unfortunately not possible in this study.

Second, for high positive S (steel ball running faster than the disc) the bulk heating of the steel ball is greater $[5,18]$. In addition, the characteristics of the test rig need to be taken into account. The steel shaft connected to the specimen is supported by two ball bearings acting like an additional heat source for high rotational speeds. For $\mathrm{S}>0$ the shaft is rotating fast in order to reach the highest tested surface velocities (especially when using a small specimen, as in the present work). These effects lead to an increase of the steel ball temperature. At high slide-to-roll ratios like $S=|1.8|$ the 
increase of the steel ball temperature is probably outweighed by the internal heating in the contact region. The temperature measurement by means of an infrared technique has been studied by Yagi, et al. $[15,16]$. It has been shown that the oil film temperatures at high slide-to-roll ratios are significantly higher than ball surface temperatures. However, both effects (bulk heating and test rig characteristics) must be taken into account for small and intermediate slide-to-roll ratios, like $\mathrm{S}=|0.8|$. For example, looking at Fig. 6 , it can be seen that the friction coefficient decreases significantly for $\mathrm{S}=+0.8$ at higher velocities. The increase in the steel ball temperature at higher velocities could heat the oil in contact. Therefore, the decrease in the friction coefficient for higher velocities is less pronounced for $\mathrm{S}=-0.8$ (Fig. 3 ) when the shaft does not rotate as fast as for $\mathrm{S}=+0.8$.

The slide-to-roll ratio of $S=-0.8$ is not high enough that the effect of the thermal viscosity wedge action leads to a significant increase in oil temperature as explained for $\mathrm{S}=-1.8$. In other words, $\mathrm{S}=$ 0.8 operates at conditions close to the oil test temperature $\left(\mathrm{T}=12^{\circ} \mathrm{C}\right)$, whereas for $\mathrm{S}=+0.8$ a higher increase in oil temperature is caused by the high speed of the shaft and the bulk heating, which in turn leads to an increase of the steel ball temperature. If $\mathrm{S}=-1.8$, a higher increase in oil temperature is caused by the temperature viscosity wedge action.

The authors assume that repeating the same experiments for higher oil temperatures, or using different oils having a viscosity less sensible to temperature variations, will reduce the possibility of an intersection in the friction coefficient trends or at least reduce the influence of the test rig characteristics. Further investigation with different oils and test temperatures are necessary.

\subsection{Film thickness}

The explanations for the general decrease in film thickness for higher $|\mathrm{S}|$ and the asymmetry in the film thickness plots over S shown in Fig.10 are similar to the ones given for the friction coefficient. 
The central film thickness apparently diminishes more for positive slide-to-roll ratios. As shown also in $[7,11]$, at high positive values of S the EHL contact appears flattened compared to high negative values, where a large difference between minimum and central film thickness emerges. It must be annotated that this asymmetry seems to occur only in contacts between materials with different thermal properties [7].

Different explanations mostly treated separately can be found in literature. The authors believe that different effects could act contemporaneously: thermal effects outside the contact and within the contact are coming together, whereas the effects within the contact could be more responsible for the asymmetry in the film thickness values for different $|S|$. One thermal effect outside the contact is the inlet heating.

In [3] a thermal correction factor is proposed. The correction factor is a function of $\mathrm{S}$ and a thermal loading parameter L, defined as:

$$
L=\frac{\beta \eta_{0} u_{e}^{2}}{\kappa}
$$

where $\beta$ is the temperature viscosity coefficient and $\kappa$ the thermal conductivity. If $L<0.1$ then thermal effects are said to be negligible. In this work for an entraining velocity of about $\mathrm{u}_{\mathrm{e}}=0.25 \mathrm{~m} / \mathrm{s}$ a loading parameter of $\mathrm{L}=0.018$ was calculated. Thus the effect of inlet heating should be very small. Nevertheless, applying the correction factor defined by Wilson in [3] also for small L a significant reduction in the film thickness was calculated for high $|\mathrm{S}|$, albeit less than the measured one. However, this cannot explain the asymmetry.

Some explanations are given in [5] for the decrease and also the asymmetry in film thickness for high $|\mathrm{S}|$, although some phenomena are not fully clear. As already explained in section 5.1, bulk heating of the steel ball is greater when it slides faster than the disc, i.e. for $\mathrm{S}>0$. Furthermore, as 
reported in [20], when $S<0$, the speed of the surface with the smaller thermal effusivity (glass) is higher than that with the greater thermal effusivity (steel). When $S>0$, the speed of the surface with the greater thermal effusivity (steel) is higher than that with the smaller thermal effusivity (glass). Thus, the heat transfer conditions are not symmetrical although the speed conditions are. In [5] also the development of a slip plan within the contact is reported. The slip plane divides the lubricant film into two layers. The position of the slip plane, which defines the decrease of the film thickness, depending on $\mathrm{S}$, is calculated by flash temperature theory and leads to the asymmetric film thickness behavior.

Another thermal effect within the contact may be related to the temperature-viscosity wedge action as explained above. For the tests carried out here, this explanation is convincingly, because it could also explain to some extent the behavior of the friction coefficients. Furthermore, as characteristic for the temperature viscosity wedge action, a relatively flat EHD contact is found for high positive $\mathrm{S}$ and a bigger difference in central and minimal film thickness for high negative $\mathrm{S}$.

The time-dependent film thickness behavior found under changing velocity is related to the time needed by the fluid to enter and escape the contact zone. For instance when accelerating, the film thickness is lower compared to stationary speed conditions. That is because the entrapped quantity of oil is less compared to the quantity under stationary conditions at same instantaneous speed conditions. In other words, the lubricant has been dragged at lower speed in the EHD contact. The tests carried out in this work never reach zero velocity and produce therefore a combination of transient hydrodynamic and squeeze motion. Pure squeeze conditions (corresponding to zero entraining velocity) do not occur, but a squeeze effect is always present connected to a film thickness variation with time.

Another interesting effect was found under transient conditions. Looking on the film thickness loops presented in Fig.11 and Fig.12 a kind of dependence between the loops and the slide-to-roll ratio occurs. For pure rolling conditions $(S=0)$ the film thickness loops are bigger than for high 
slide-to-roll ratios. Furthermore, for high negative $\mathrm{S}$ the film thickness loops do not reduce as much as for positive S. As more and more non-metallic materials will be introduced in mechanical engineering, a detailed knowledge of this effect might help in optimizing non-conformal lubricated machine elements. A first explanation could be that the squeeze effect is reinforced for negative $\mathrm{S}$ by the previously described variation of the difference between minimum and central film thickness in dependence of the slide-to-roll ratio. Fig. 15 presents an example of the central zone appearing as a deep cavity between the side lobes for a negative slide-to-roll ratio, while it is relatively flat for the corresponding positive value.

\subsection{Friction coefficient - film thickness relation}

The above discussion can explain to some extend the behavior of the friction and film thickness trends. However, it is always difficult to find direct correlations between film thickness values and friction coefficients. For example, looking at Fig.10, even though the central film thickness is reduced significantly for higher velocities and positive high S, the friction coefficients in Fig.6 do not show big differences (compare for instance the curves for $S=+0.8$ and $S=+1.8$ for $u_{e}>0.15 \mathrm{~m} / \mathrm{s}$ ).

Looking at the results obtained under transient conditions, it seems even more difficult to find a relation. The film thickness loop reduction found by increasing S (Fig.11 and Fig.12) does not correspond to a reduction of the friction loops (see for instance Fig.8). Probably squeeze, thermal and geometrical aspects combined together give results very difficult to be interpreted. Also possible vibration problems and the methodology used for the analysis of the recorded friction data can have a certain influence on the results as shown in [19]. There, friction coefficient loops of diminishing amplitude by increasing $\mathrm{S}$ were found. However, the roughness of the specimens in [19] was higher, the viscosity of the lubricant lower and therefore the lambda ratio smaller than in the present work. For higher S and thus increasing shearing, the film thickness will decrease additionally. This leads to a greater amount of asperity contacts that could reduce the effect of 
squeeze and amplitude of the loops as reported in [10]. In this work, however, also for high $\mathrm{S}$ the influence of roughness is relatively small. Therefore, the variation of the height of the film thickness alone is not sufficient to explain the behavior of the friction coefficient and different effects must be taken into account as explained above.

\section{CONCLUSIONS}

From the results presented and discussed the following main conclusions can be drawn:

1. The slide-to-roll-ratio has an influence on the EHD friction coefficients under steady state as well as transient conditions. While higher $|\mathrm{S}|$ causes normally higher friction coefficients, an intersection for high and negative $\mathrm{S}$ was found that produce a reduction in friction values. This has been confirmed also by testing steel balls of different diameter. A possible explanation can be the temperature viscosity wedge action and the low oil temperature used. The thermal effects on the friction coefficients found for steady state conditions are superimposed by transient effects when changing the velocity with a sinusoidal law.

2. At high $\mathrm{S}$ and steady state conditions the film thickness falls from the value measured under pure rolling $(\mathrm{S}=0)$ in an asymmetric way. This means the central film thickness reduces more for positive S by around $30 \%$, whereas for negative S by about $20 \%$. Under transient conditions a similar behavior is found independently on the test frequency. One possible explanation for the asymmetric behavior in film thickness reduction could be the temperature viscosity wedge action. A second explanation, which does not contradict the first one, could be the greater bulk heating of the steel ball when it slides faster than the disc as reported in $[5,20]$. 
3. Under transient conditions high slide-to-roll ratios seem to reduce the squeeze effect on the film thickness. This effect is more pronounced for higher test frequencies. The reduction of the film thickness loops is higher for positive $\mathrm{S}$ than for negative $\mathrm{S}$. A possible explanation for this difference could be that the temperature viscosity wedge action found for negative $\mathrm{S}$ amplifies the squeeze effect. In this case, such effect should not be found when contacting materials with the same thermal properties are used. This means for example in a glass-onglass or steel-on-steel contact a uniform or similar reduction of the loops is assumed for negative and positive S. An experimental verification, however, using for example a ceramic or glass ball instead of a steel ball is necessary. 


\section{References}

[1] Grubin AN, Vinogradova IE. Investigation of the Contact of Machine Components. Central Scientific Research Institute for Technology and Mechanical Engineering, Book 30, Moscow 1949.

[2] Dowson D, Higginson GR. Elastohydrodynamic Lubrication. Pergamon, Oxford, 1966.

[3] Wilson WRD, Sheu S. Effects of Inlet Shear Heating Due to Sliding on Elastohydrodynamic Film Thickness. ASME Journal of Lubrication Technology 1983; 105:187-188.

[4] Olver AV. Testing transmission lubricants: the importance of thermal response. Journal Aero. Eng., Proc.Instn. Mech. Engrs 1991; 205: 35-44.

[5] Smeeth M, Spikes HA. The Influence of Slide/Roll Ratio on the Film Thickness of an EHD Contact Operating Within the Mixed Lubrication Regime. Proceedings of the 22th LeedsLyon Symposium on Tribology, 1995. Oxford: Elsevier, 1996 pp. 695-703.

[6] Kaneta M. For the Establishment of a new EHL Theory. Proceedings of the 25th Leeds-Lyon Symposium on Tribology, 1999. Oxford: Elsevier, pp. 25-36.

[7] Kaneta M, Yang P. Effects of Thermal Conductivity of Contacting Surfaces on Point EHL Contacts. ASME J.o.T. 2003; 125:731-738.

[8] Sugimura J, Jones WR, Spikes HA.EHD Film Thickness in Non-Steady State Contacts. ASME J.o.T. 1998; 120:442-452.

[9] Zhao J, Sadeghi F. Analysis of EHL Circular Contact Shut Down. ASME J.o.T. 2003; 125:7690.

[10] Zhai X, Needham G, Chang L. On the Mechanism of Multi-Valued Friction in Unsteady Sliding Line Contacts Operating in the Regime of Mixed-Film Lubrication. ASME J.o.T. 1997; 119:149-155.

[11] Bassani R, Ciulli E, Carli M, Stadler K. Experimental investigation of transient and thermal effects on lubricated non-conformal contacts, Tribotest 2007; 13: 183-194.

[12] Larsson R, Larsson PO, Eriksson E, Sjöberg M, Höglund M. Lubricant properties for input to hydrodynamic and Elastohydrodynamic lubrication analyses. Proc Instn Mech Engrs 2000; Part J, 214:17-27. 
[13] Ciulli E, Draexl T, Stadler K. Film Thickness Analysis for EHL Contacts under Steady-State and Transient Conditions by Automatic Digital Image Processing", Advances in Tribology, vol. 2008, Article ID 325187, 16 pages, 2008. doi:10.1155/2008/325187.

[14] Bassani R, Ciulli E, Stadler K, Carli M. Lubricated non-conformal contacts under steady state and transient conditions. Proceedings of the AIMETA general conference, Florence 2005.

[15] Yagi K, Kyogoku K, Nakahara T. Mechanism of Dimple Formation under Elastohydrodynamic Conditions. Proceedings of the 29th Leeds-Lyon Symposium on Tribology, 2002. Oxford: Elsevier, 2003 pp. 111-120.

[16] Yagi K, Kyogoku K, Nakahara T. Temperature measurements of oil film and surface in point contact EHL under high slip ratio conditions. Proc. of the Int. Tribology Conf. Nagasaki, 2000. pp. 567-572.

[17] Chang L, Qu S, Webster MN, Jackson A. On the mechanisms of the reduction in EHL traction at low temperature. Tribology Transaction 2006; 49:182-191.

[18] Jaeger, JC. Moving surfaces of heat and temperature at sliding contact. Proc.Roy.Soc.New.S.Wales 1942; 76:203-204.

[19] Ciulli E. Time and frequency domain analysis of experimental EHL transient conditions data. $11^{\text {th }}$ Nordic Symposium on Tribology, Proceedings of the Nordtrib 2004, pp. 575-584.

[20] Yagi K, Kyogoku K, Nakahara T. Relationship between temperature distribution in EHL film and dimple formation. ASME J.o.T. 2005; 127:658-665. 


\section{Figure captions}

Fig.1: Picture of the test rig used.

Fig.2: Friction coefficient as a function of speed for different negative (a) and positive (b) values of S under steady state conditions. Spherical specimen with a diameter of $15.081 \mathrm{~mm}$.

Fig.3: Friction coefficient as a function of speed under steady state condition; $\mathrm{S}<0$.

Fig.4: Friction coefficient as a function of speed under transient condition; test frequency of $0.25 \mathrm{~Hz}, \mathrm{~S}<0$.

Fig.5: Friction coefficient as a function of speed under transient condition; test frequency of $1 \mathrm{~Hz}$, $\mathrm{S}<0$.

Fig.6: Friction coefficient as a function of speed under steady state condition; $\mathrm{S}>0$.

Fig.7: Friction coefficient as a function of speed under transient condition; test frequency of $0.25 \mathrm{~Hz}, \mathrm{~S}>0$.

Fig.8: Friction coefficient as a function of speed under transient condition; test frequency of $1 \mathrm{~Hz}$, $\mathrm{S}>0$.

Fig.9: Central film thickness $h_{\text {cen }}$ as a function of speed under steady state conditions for $S<0$ (a) and $\mathrm{S}>0$ (b).

Fig.10: Central film thickness $\mathrm{h}_{\text {cen }}$ as a function of $\mathrm{S}$ for different velocities. 
Fig.11: Film thickness for pure rolling conditions. $\mathrm{h}_{\mathrm{cen}}$ for $1 \mathrm{~Hz}$ and steady state conditions (single line) (a); $\mathrm{h}_{\text {cen }}$ (thick lines) and $\mathrm{h}_{\min }$ (thin lines) for $0.25 \mathrm{~Hz}$ (dashed lines) and $1 \mathrm{~Hz}$ (solid lines) (b).

Fig.12: Central (thick lines) and minimum (thin lines) film thickness for $0.25 \mathrm{~Hz}$ (dashed lines) and $1 \mathrm{~Hz}$ (solid lines) for negative and positive values of $\mathrm{S}: \mathrm{S}=-1.8$ (a), $\mathrm{S}=-0.8$ (b), $\mathrm{S}=+1.8$ (c) and $\mathrm{S}=+0.8(\mathrm{~d})$.

Fig.13: Central film thickness $\mathrm{h}_{\text {cen }}$ over time compared to the entraining velocity for $1 \mathrm{~Hz} ; \mathrm{S}=-1.8$ (a), $\mathrm{S}=0$ (b) and $\mathrm{S}=+1.8$ (c).

Fig.14: Monochromatic interferograms for different $\mathrm{u}_{\mathrm{e}}$ and $\mathrm{S}=-1.8$ (entraining of the oil from the left).

Fig.15: Comparison of mid-plane profile perpendicular to rolling direction for positive and negative $\mathrm{S}$ and $\mathrm{u}=0.2 \mathrm{~m} / \mathrm{s}$. 


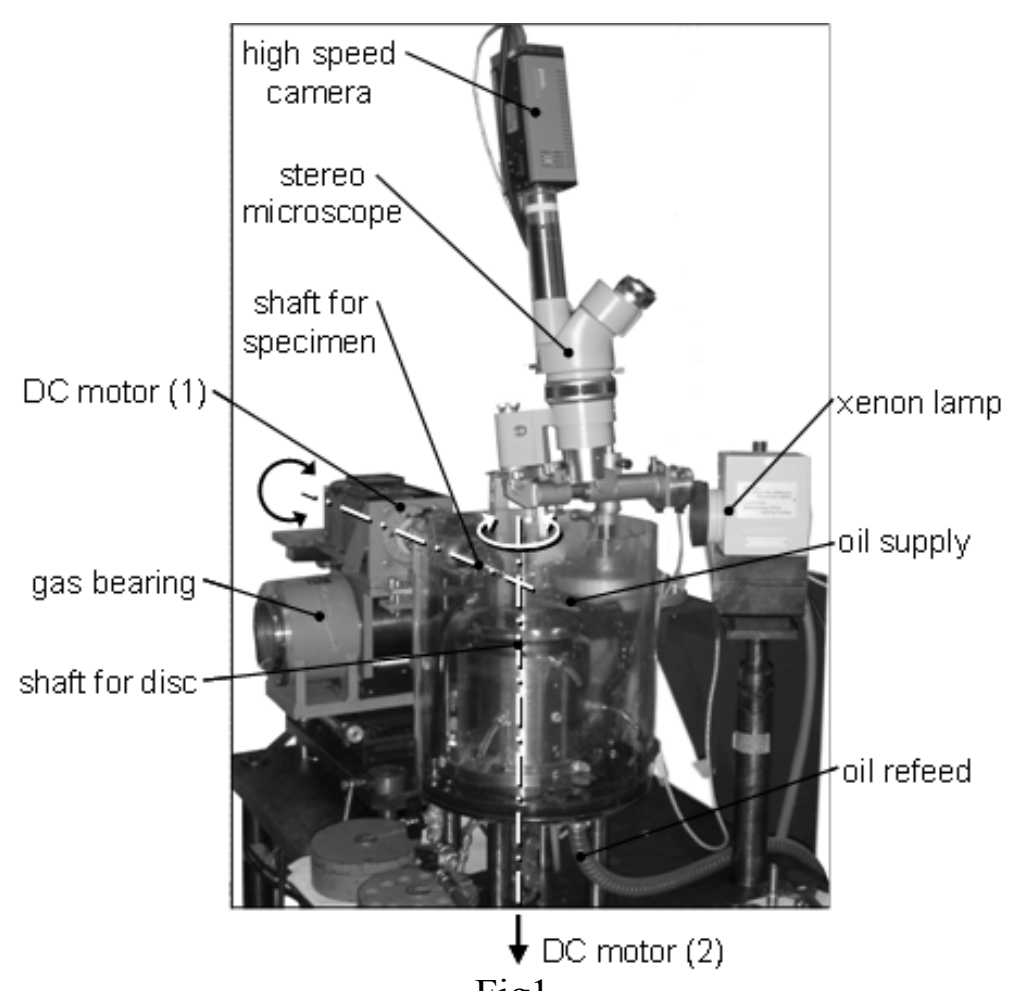

Fig1
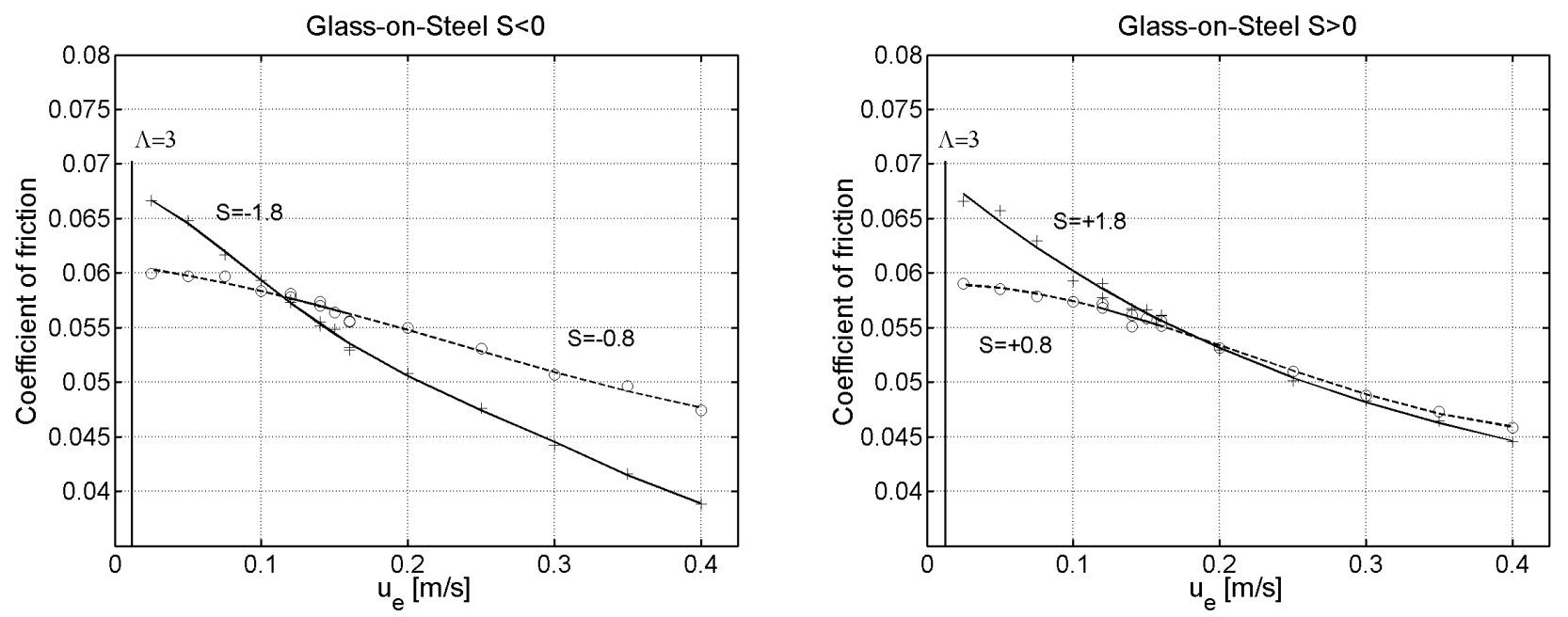

Fig.2

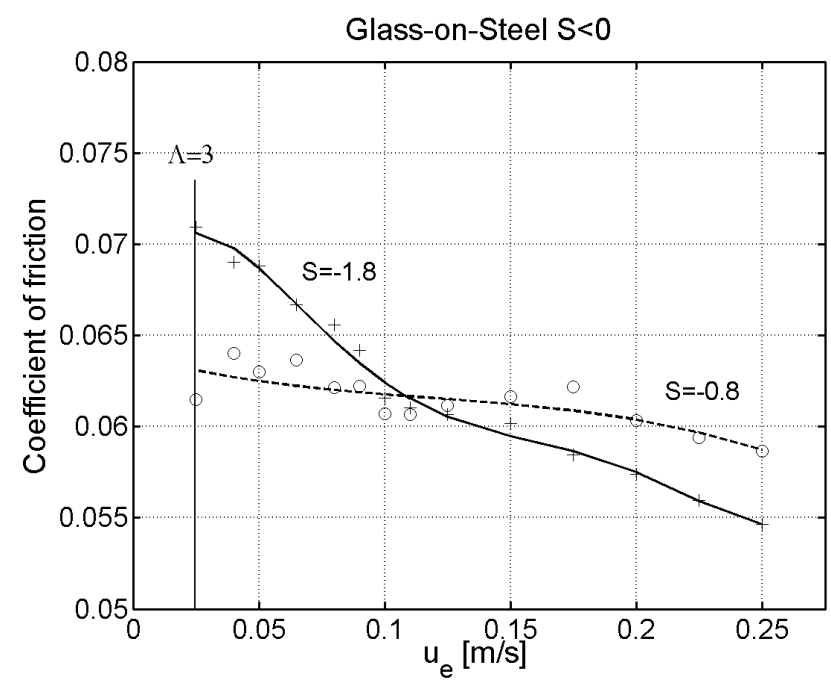

Fig. 3 


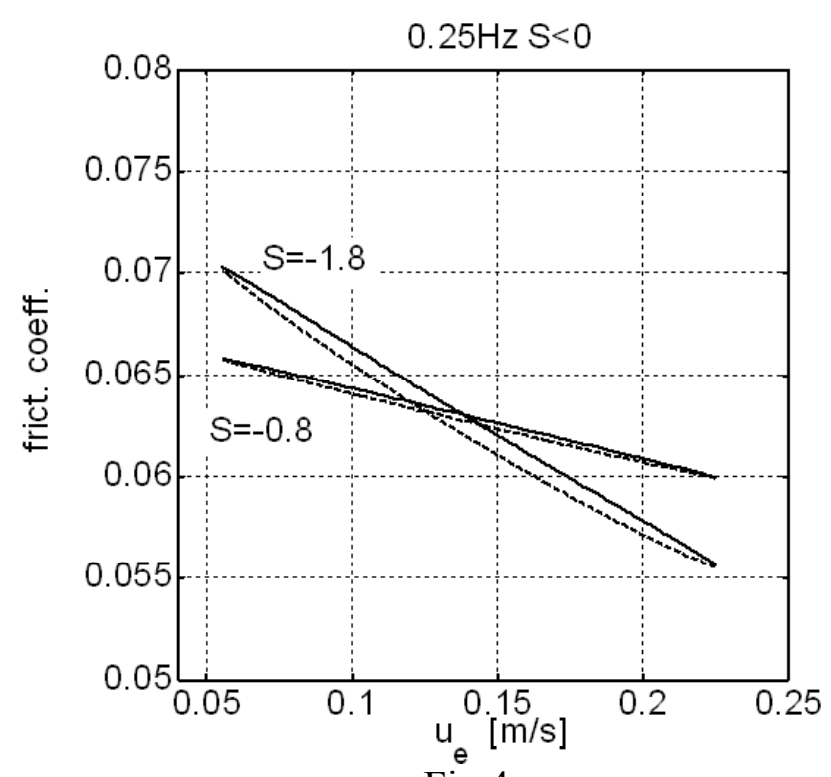

Fig.4

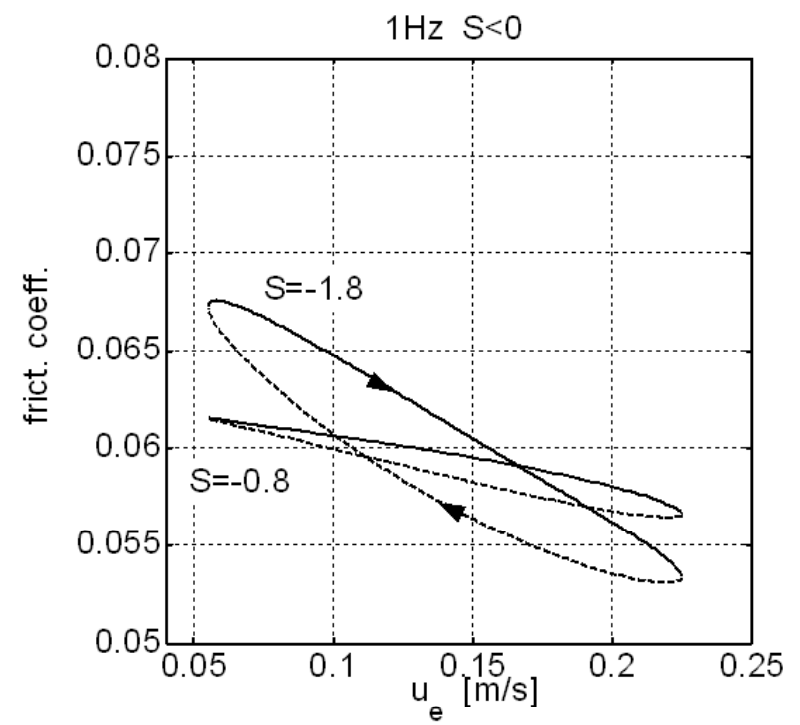

Fig.5

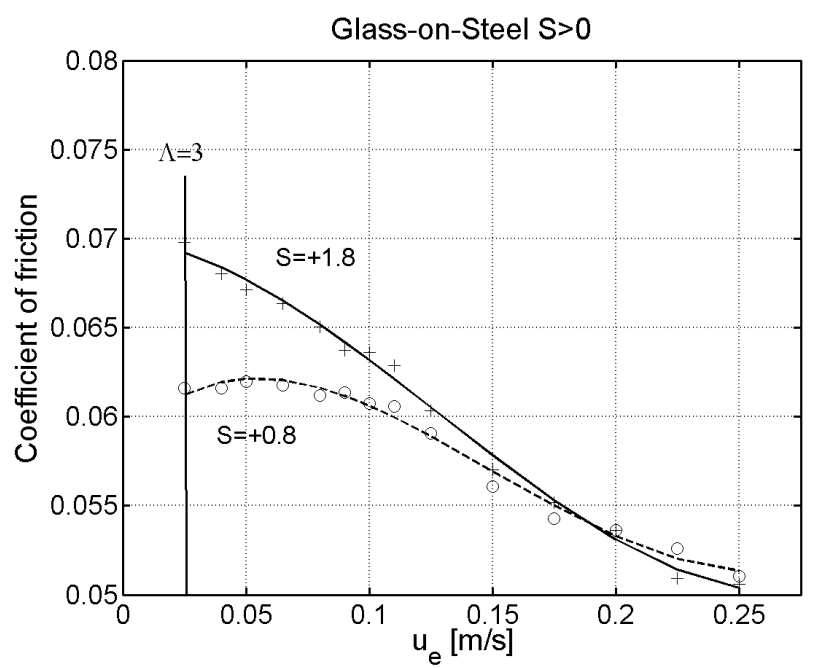

Fig.6 


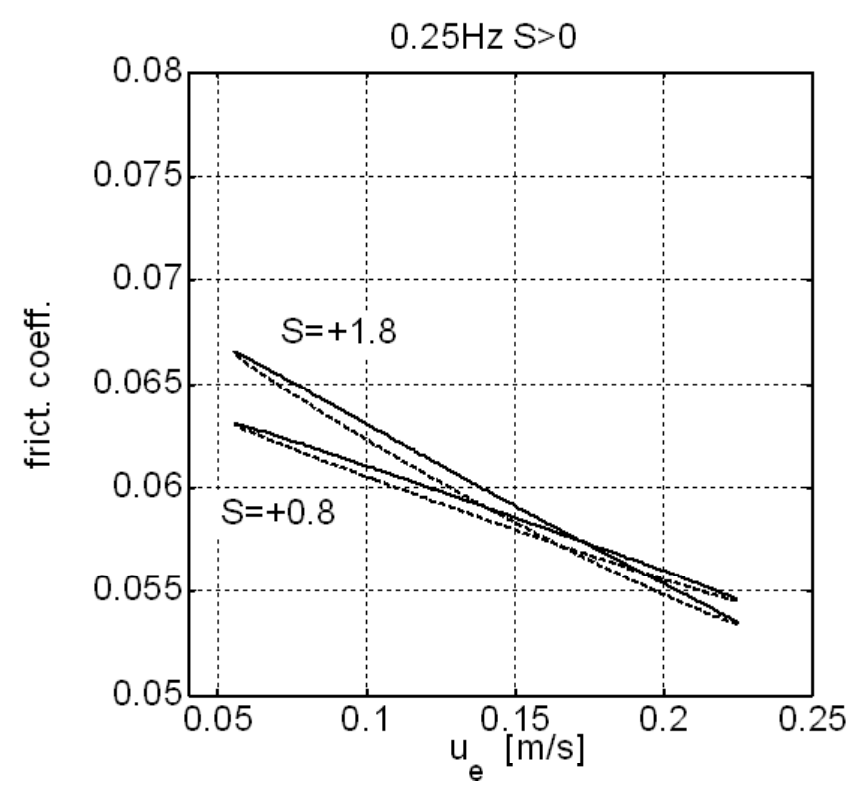

Fig.7

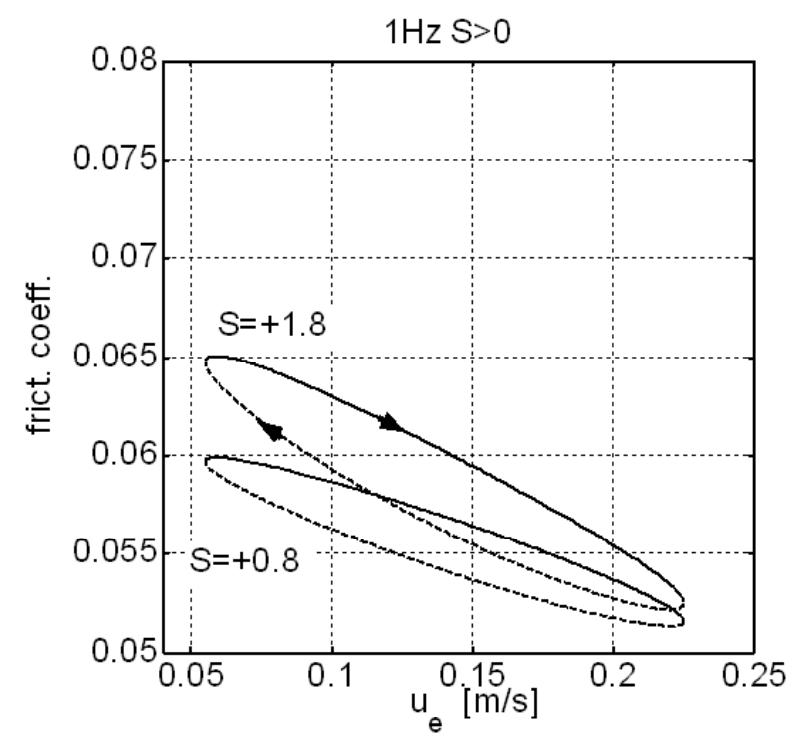

Fig. 8
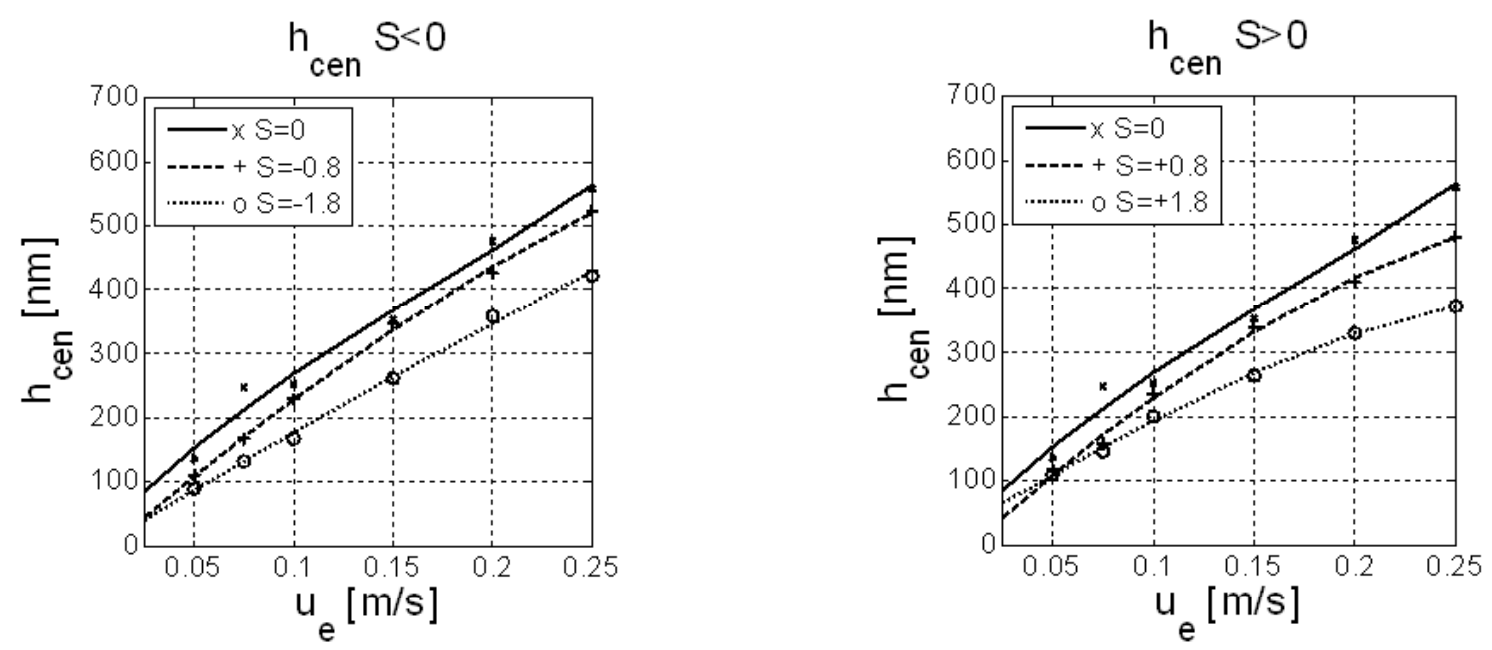

Fig.9 


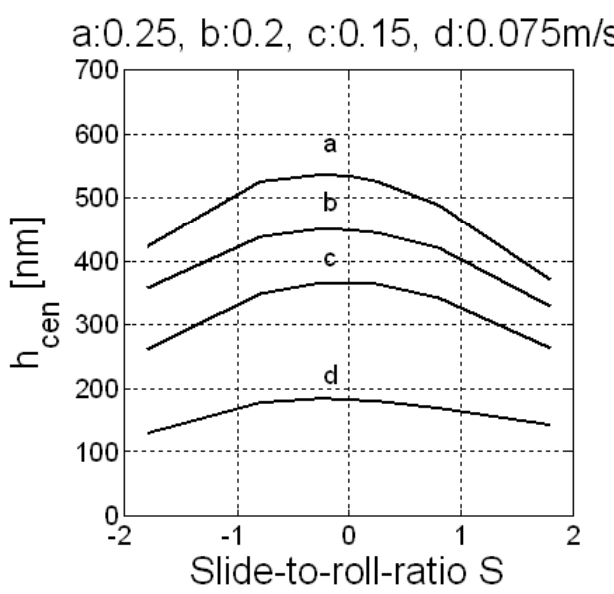

Fig. 10
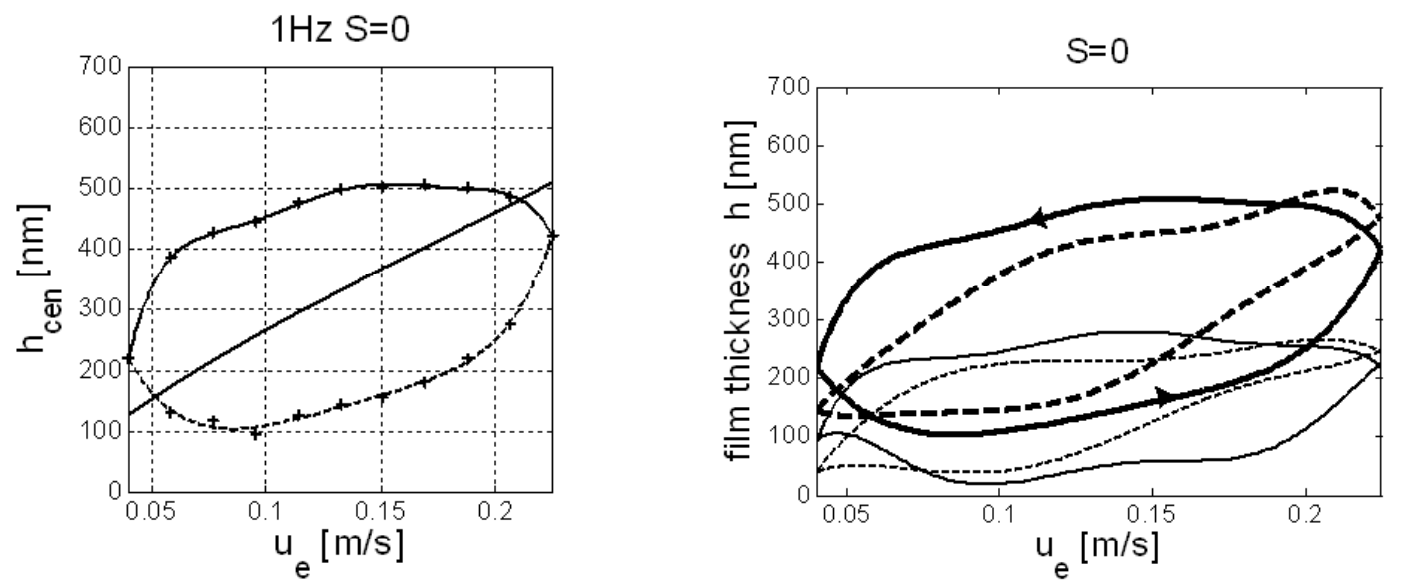

Fig.11
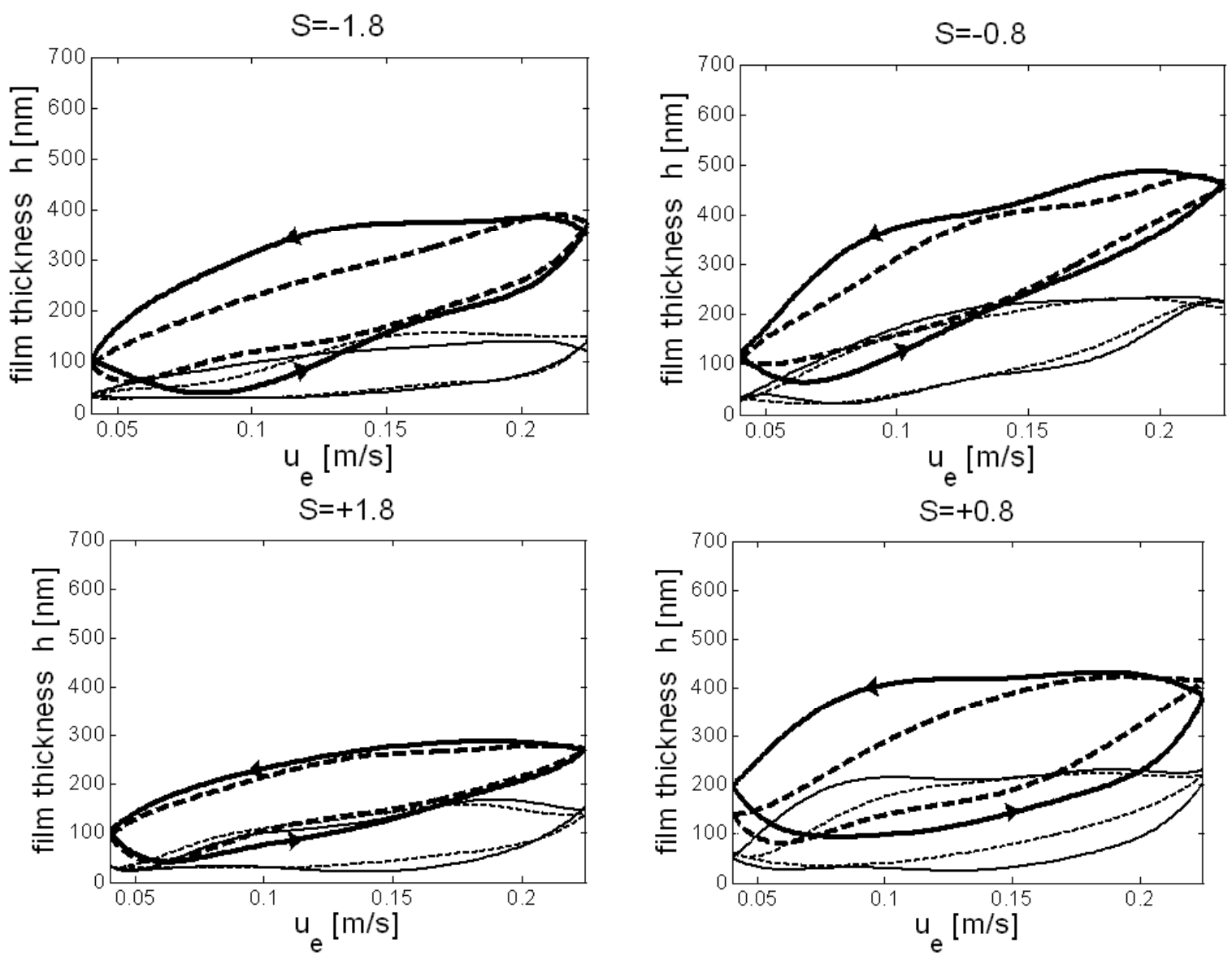

Fig. 12 

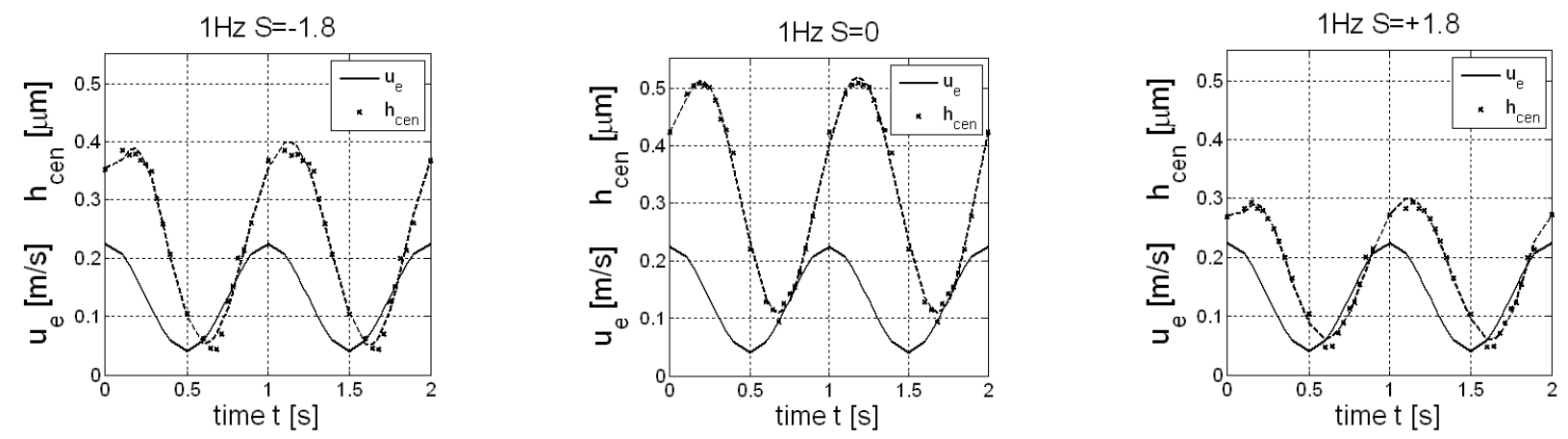

Fig.13

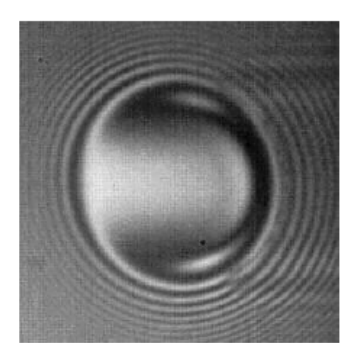

$\mathrm{u}_{\mathrm{e}}=0.15 \mathrm{~m} / \mathrm{s}$

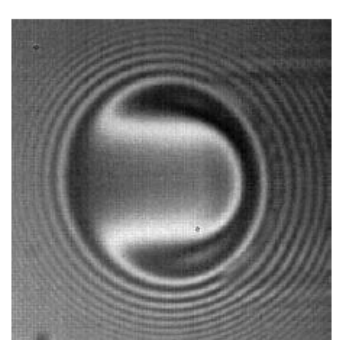

$\mathrm{u}_{\mathrm{e}}=0.2 \mathrm{~m} / \mathrm{s}$

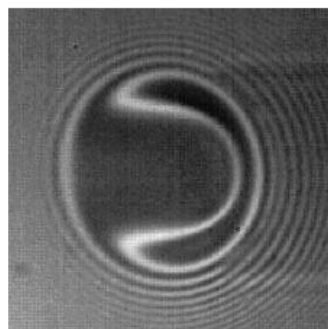

$\mathrm{u}_{\mathrm{e}}=0.25 \mathrm{~m} / \mathrm{s}$

Fig. 14

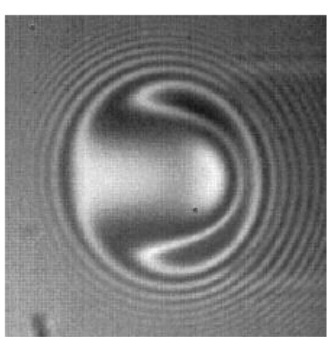

$\mathrm{u}_{\mathrm{e}}=0.3 \mathrm{~m} / \mathrm{s}$

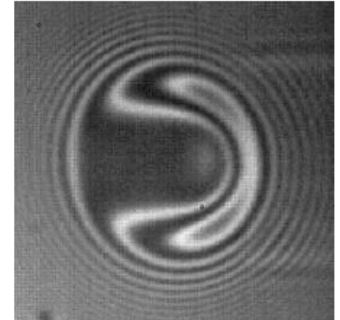

$\mathrm{u}_{\mathrm{e}}=0.4 \mathrm{~m} / \mathrm{s}$

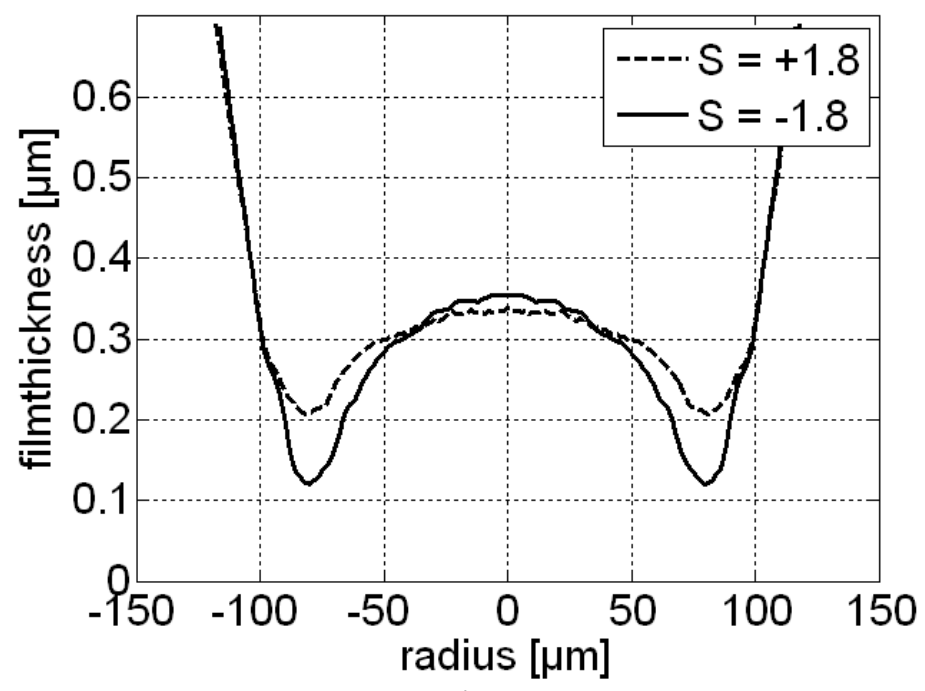

Fig. 15 